\title{
Ornamental Asteraceae Species as New Sources of Secondary Metabolites
}

\author{
Ana Flavia Burlec ${ }^{1}$, Cristina Arsene ${ }^{2}$, Elvira Gille ${ }^{3}$, Monica Hăncianu ${ }^{1}$, Oana Cioancă ${ }^{1}$ \\ ${ }^{1}$ Department of Pharmacognosy, Faculty of Pharmacy, "Grigore T. Popa" University of Medicine and Pharmacy, lasi, ROMANIA. \\ 'Department of Biochemistry, Faculty of Medicine, "Grigore T. Popa" University of Medicine and Pharmacy, lasi, ROMANIA. \\ ${ }^{3}$ The National Institute of R \& D for Biological Science Bucuresti/ the "Stejarul" Biological Research Centre, Piatra Neamt, ROMANIA.
}

\begin{abstract}
Objective: This paper presents the results regarding the morphological characters, the content in total phenols, the metabolites profile and the antioxidant activity of outdoor cultivated Rudbeckia hirta var. Goldilocks and Tagetes erecta plants. Material and Methods: The plant material was represented by flower buds and fully bloomed inflorescences taken from Rudbeckia hirta var. Goldilocks and Tagetes erecta. Morphological assessment was carried using microscopy, while chemical assessment used thin layer chromatography (TLC) and UHPLC methods. The total phenolic content was established with FolinCiocalteu method. The antioxidant activity was evaluated with DPPH radical scavenging tests. The secondary metabolites were evaluated in two different nutritional statuses. Results: The results indicated that the studied ornamental Asteraceae species represent rich sources of phenols. However, the fertilization lowers the plant's biosynthetic capacity for polyphenols for both Rudbeckia and Tagetes flowers. Thus, by fertilizing, the flowers of Tagetes lose components such as polyphenolic acids, modifying their spectrum. Regarding the antioxidant activity, Tagetes erecta showed a promising activity against free radicals. Conclusion: The concentration of plant secondary metabolites was found to be influenced by the nutritional status. Both species represent a good source for secondary metabolites, but their biological potential remains to be established.
\end{abstract}

Key words: Rudbeckia, Tagetes, Secondary metabolites, Ornamental plants, Antioxidant activity.

\section{INTRODUCTION}

A very large number of secondary metabolites from plants have been described until now. ${ }^{1}$ Secondary metabolites do not possess fundamental roles in the plant metabolism, but serve different important functions in the plant such as protection against herbivores and harmful microorganisms. ${ }^{2}$ They have been divided in several categories, taking into consideration their chemical structure and the presence or absence of different chemical elements such as nitrogen and oxygen. ${ }^{1}$ Most plant secondary metabolites do not contain nitrogen and in this category important bioactive compounds have been described. Different classes of constituents such as flavonoids, polyphenolic acids and terpenoids were established among nitrogen-free compounds. ${ }^{1}$ Some of these constituents have been associated with a series of potential therapeutic actions, showing good antioxidant, antibacterial or anti-inflammatory activities. ${ }^{3,4}$ There is still the need for discovering new sources rich in such compounds that could facilitate their use in the pharmaceutical and food industries. The Asteraceae family is one of the largest families and comprises of many therapeutic, edible and ornamental species. ${ }^{5}$ The latter group has been seldom investigated regarding chemical composition and could represent a new category of sources of secondary metabolites with important biological activity.
DOI: 10.5530/ijper.51.3s.61 Correspondence: Hăncianu Monica, Department of Pharmacognosy, Faculty of Pharmacy, Grigore T. Popa" University of Medicine and Pharmacy, Iasi, ROMANIA

Contact: +40232301815 E-Mail: mhancianu@yahoo. com 


\section{MATERIAL AND METHODS}

The plant material was represented by flower buds (f1) and fully bloomed inflorescences (f2) taken from Rudbeckia hirta var. Goldilocks (black-eyed-Susan) and Tagetes erecta (Mexican marigold) cultivated in northeastern Romania in the year of 2015. Voucher specimens are deposited with no. 2015/Rf and 2015/Tf at the Department of Pharmacognosy ("Grigore T. Popa" University of Medicine and Pharmacy Iasi) for ready reference. The secondary metabolites were evaluated in two different nutritional statuses, unfertilized (V1) and fertilized (V2) with Osmocote $\left(75 \mathrm{~g} / \mathrm{m}^{2}\right)$. Chemical assessment was carried on dry methanolic extracts $(1: 4 \mathrm{v} / \mathrm{v})$ obtained through sonication extraction for 30 minutes. The dry extracts were diluted in $1 \mathrm{~mL}$ methanol for qualitative determination of phenolic compounds using thin layer chromatography (TLC), while for UHPLC assay the extracts were prepared by dissolution of each dry extract in acetonitrile. The chemical profile of flowers was investigated for polyphenolic acid derivatives, flavonoids and alantolactone. The total phenolic content was established with Folin-Ciocalteu method, ${ }^{6}$ using gallic acid as standard. The results were expressed as Gallic acid equivalents (GAE)(mg/ 100g dry extract). Semi quantitative analyses of alantolactone were performed using the HPLC method described by Ivănescu (2015) with modifications. ${ }^{7}$ The method was adapted for a ThermoUltiMate3000 gradient chromatograph controlled by Chrome Leon interface, an auto sampler, an Accucore XL C18 column (150x4, 6x4) and multidiode array detector (DAD). The antioxidant activity was established with DPPH (1,1-diphenyl-2-picrylhydrazyl) radical scavenging assay, ${ }^{8}$ one of the most widely used antioxidant test for plant extracts. The absorption was measured at $517 \mathrm{~nm}$ for 5 minutes and gallic acid was used as standard.

\section{RESULTS AND DISSCUSSION}

The morphological features were confirmed by microscopical analysis that indicated the presence of the main elements that characterize the investigated species: pollen grains, surface hairs, glandular trichomes, flower epidermis, papillae.

The results indicated that the studied ornamental Asteraceae species represent rich sources of phenols. However, the fertilization lowers the plant biosynthetic capacity for polyphenols for both Rudbeckia (1146 mg GAE/100g V1f2 dry extract, compared to $871.9 \mathrm{mg}$ GAE/100g V2f2 dry extract) and Tagetes flowers (526.62 mg GAE/100g V1f2 dry extract compared to V2f2, 428.55 mg GAE/100g dry extract). Generally, total phenols are important for plant defense against UV rays and harsh environmental conditions. However, as others have observed the fertilization (V2) with $\mathrm{N}$ and $\mathrm{P}$ reduced the plant chemical defense, although no other changes were made to our crops. ${ }^{9}$ Nevertheless, the ornamental properties of both species increased significantly with fertilization, both biomass, the number and diameter of inflorescences were greater in the fertilized samples (V2).${ }^{10}$ The TLC analysis indicated qualitative differences between the phenophases (f1 and f2) and less important changes between the fertilized samples. The standard (STD) used for TLC was represented by a mix of phenol carboxylic acids and flavonoids (chlorogenic acid, hyperoside, and rutoside). The blue spots indicate the presence of phenol carboxylic acids, while orange spots point out the presence of flavonoids. The flower buds and the fully bloomed inflorescences of Rudbeckia birta var. Goldilocks contain chlorogenic acid $\left(\mathrm{R}_{\mathrm{f}} 0,47\right)$ and hyperoside $\left(\mathrm{R}_{\mathrm{f}} 0,51\right)$.

Caffeic acid was also present in both fertilized and unfertilized fully bloomed inflorescences, but not in flower buds. The flowers buds and the fully bloomed inflorescences of Tagetes erecta contain isochlorogenic acid and ferulic acid. The first can be found in higher concentrations in the unfertilized samples, while ferulic acid can be found in higher concentrations in the fertilized ones. Caffeic acid and chlorogenic acid are also present in both phenophases, but in lower quantities than the other two phenol carboxylic acids. A luteolin glycoside ( $\operatorname{Rf} 0,60)$ can also be found in most extracts, except the one obtained from fertilized fully bloomed inflorescences.

Terpenoid compounds were also found to be important components of these species, indicating possible implications in allergic reactions. Alantolactone was found in Rudbeckia flower buds $(0,2358 \mu \mathrm{g} / 100 \mathrm{~g}$ dry flowers), its quantity decreasing in the unfertilized plants. On the other hand, alantolactone was found in both phenophases of Tagetes flowers, the fertilized fully grown flowers presenting the highest content $(0,2459 \mu \mathrm{g} / 100 \mathrm{~g}$ plant material).

The antioxidant activity of the samples was investigated as the scavenging capacity towards the DPPH radical. In such reactions, a good scavenger changes the violet solution to yellow, indicating the existence of antioxidant potential. ${ }^{11}$ In the present study, both Rudbeckia hirta and Tagetes erecta extracts had good antioxidant activity (Table 1).

However, the Mexican marigold showed a lower value of EC50 which is equivalent to a better antioxidant potential. Even though the total polyphenolic content is higher for black-eyed-Susan, Mexican marigold 


\begin{tabular}{|c|c|}
\hline \multicolumn{2}{|c|}{ Table 1: Results of the DPPH assay } \\
\hline Samples & $\mathrm{EC}_{50}(\mathrm{mg} / \mathrm{ml})$ \\
\hline Rudbeckia hirta var. Goldilocks extract & 6.54 \\
\hline Tagetes erecta extract & 0.76 \\
\hline Gallic acid & 0.027 \\
\hline
\end{tabular}
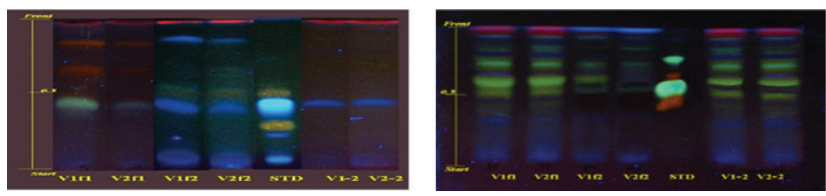

Figure 1: TLC chromatogram for polyphenols of Rudbeckia hirta (left) and of Tagetes erecta extracts (right)
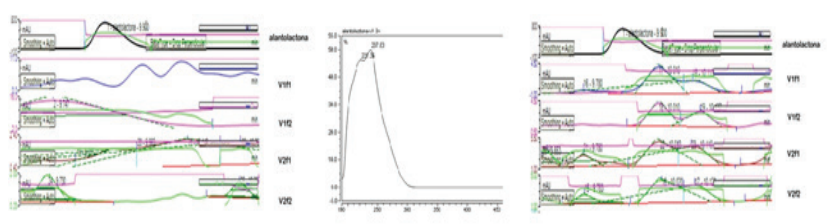

Figure 2: HPLC chromatogram for alantolactone in the investigated samples.

Rudbeckia hirta var. Goldilocks extracts (left), UV spectra of alantolactone (standard), HPLC chromatogram for alantolactone in Tagetes erecta samples (right); V1f1 - unfertilized flower buds; V2f1 - fertilized flower buds; V1f2 - unfertilized fully bloomed inflorescence; V2f2 - fertilized fully bloomed inflorescence.

is known to possess many other constituents such as carotenoids, that are responsible for a good scavenging activity. ${ }^{12}$ Moreover, these compounds (lutein, xanthophylls and carotenoids) are usually unstable to light and the plant stores them in organs little exposed to the sun. This may partially explain the greater intensity of yellow spots (TLC) in sepals and stems and leaves, while mature inflorescences lack or are very weak in intensity. Nevertheless, such compounds are important for plant protection against UV and other environmental stress factors. Therefore, further research is necessary to formulate a conclusive hypothesis regarding scavenging potential and carotenoid concentration.

\section{CONCLUSION}

The concentration of secondary metabolites from the investigated species was found to be influenced by the nutritional status. The obtained values indicated that the studied ornamental Asteraceae species might represent rich sources of polyphenols. Both species represent reliable sources for secondary metabolites, but their biological potential remains to be established.

\section{ACKNOWLEDGEMENT}

None

\section{CONFLICT OF INTEREST}

The authors declare that there is no conflict of interest regarding the publication of this paper.

\section{ABBREVIATION USED}

DAD: Multidiode array detector; DPPH: 1,1-diphenyl2-picrylhydrazyl; $\mathrm{EC}_{50}$ : Half maximal effective concentration; $\mathrm{f} 1$ : phenophase 1 = flower buds; $\mathrm{f} 2$ : phenophase 2 = fully bloomed inflorescences; GAE: Gallic acid equivalents; STD: Standard; TLC: Thin layer chromatography; UHPLC: Ultra High Performance Liquid Chromatography; V1: Unfertilized samples; V2: Fertilized samples.

\section{REFERENCES}

1. Kliman RM. Encyclopedia of Evolutionary Biology. Oxford: Academic Press; 2016. Wink M.

2. Thomas B, Murray BG, Murphy DJ. Encyclopedia of Applied Plant Sciences. 2nd ed. Oxford: Academic Press; 2017. Davey M.

3. Barbieri R, Coppo E, Marchese A, Daglia M, Sobarzo-Sánchez E, Nabavi SF, et al. Phytochemicals for human disease: An update on plant-derived compounds antibacterial activity. Microbiological Research 2017;196:44-68.

4. Chadwick M, Trewin H, Gawthrop F, Wagstaff C. Sesquiterpenoids Lactones: Benefits to Plants and People. International Journal of Molecular Sciences. 2013;14(6):12780-805.

5. Bessada S, Barreira J, Oliveira M. Asteraceae species with most prominent bioactivity and their potential applications: A review. Industrial Crops and Products. 2015;76:604-15.

6. Singleton VL, Rossi JA. Colorimetry of total phenolics with phosphomolybdicphosphotungstic acid reagents. American Journal of Enology and Viticulture. 1965;16(3):144-58.

7. Ivanescu B, Miron A, Corciova A. Sesquiterpene Lactones from Artemisia Genus: Biological Activities and Methods of Analysis. Journal of Analytical Methods in Chemistry. 2015:1-21.

8. Hatano T, Kagawa H, Yashura T, Okuda T. Two new flavonoids and other constituents in licorice root: their relative astringency and radical scavenging effects. Chemical and Pharmaceutical Bulletin. 1988;36(6):2090-7.

9. De Long JR, Sundqvist MK, Gundale MJ, Giesler R, Wardle DA. Effects of elevation and nitrogen and phosphorus fertilization on plant defense compounds in subarctic tundra heath vegetation. Functional Ecology. 2016;30:314-25.

10. Mircea (Arsene) C, Cioancă O, Draghia L, Hăncianu M. Morphological characteristics and polyphenol variations in Rudbeckia hirta L. Romanian Biotechnological Letters. 2015;20(4):10688-95.

11. Grădinariu VE, Cioancă $O A$, Gille EL, Aprotosoaie AC, Hritcu LU, Hăncianu MO. The chemical profile of basil biovarieties and its implication on the biological activity. Farmacia. 2013;61(4):632-639.

12. Li-wei XU, Juan CHEN, Huan-yang QI, Yan-ping SHI, Phytochemicals and Their Biological Activities of Plants in Tagetes L. Chinese Herbal Medicines. 2012,4(2):103-17. 


\section{PICTORIAL ABSTRACT}
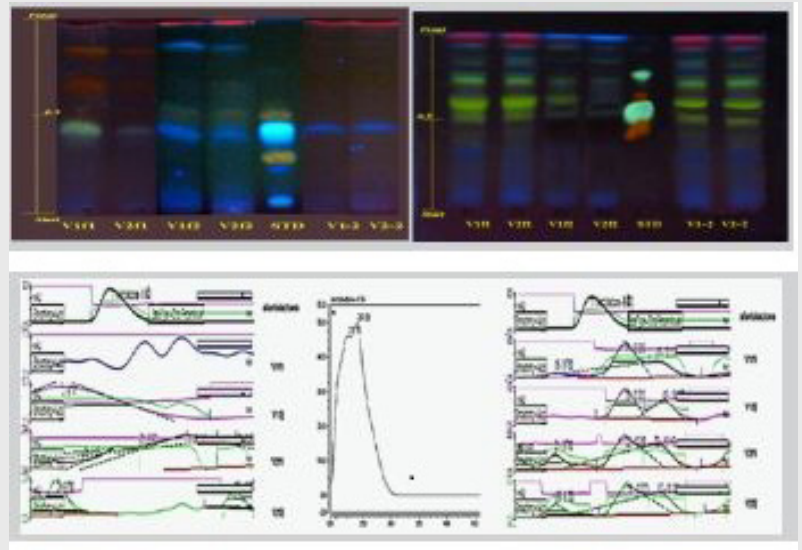

\section{ABOUT AUTHORS}

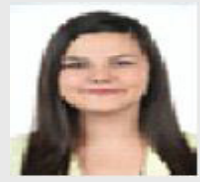

Burlec Ana Flavia: ia a PhD student with a degree in Pharmacy and residency in Clinical Laboratory.

\section{SUMMARY}

- The most important groups of secondary metabolites found in Rudbeckia hirta var. Goldilocks and Tagetes erecta plants were investigated.

- Morphological features were confirmed by microscopical analysis.

- The biosynthetic properties were investigated in different nutritional statuses (unfertilized vs. fertilized).

- The concentration of secondary metabolites was influenced by fertilization.

- Terpenoid compounds were found to be important components of the investigated species.

- Both Rudbeckia hirta and Tagetes erecta extracts had good antioxidant activity.

- The antioxidant potential is not correlated with the total phenolic content.

- The studied ornamental Asteraceae species might represent rich sources of polyphenols.

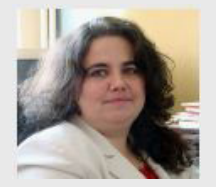

Cioanca Oana: Lecturer at Department of Pharmacognosy and Phytotherapy, Cell Biology, "Grigore T. Popa" University of Medicine and Pharmacy lasi; Pharm D, PhD in Pharmacognosy and specialist in Clinical Pharmacy; Trained into HPLC and spectrofotometry, isolation and quantification of plant bioactive compounds

Cite this article: Burlec AF, Arsene C, Gille E, Hăncianu M, Cioancă O. Ornamental Asteraceae Species as New Sources of Secondary Metabolites. Indian J of Pharmaceutical Education and Research. 2017;51(3)Suppl:S425-28. 